Article

\title{
In Vivo Wound Healing and In Vitro Anti-Inflammatory Activity Evaluation of Phlomis russeliana Extract Gel Formulations
}

\author{
Mehmet Evren Okur ${ }^{1, *}$, Ayşe Esra Karadağ ${ }^{2,3}{ }^{6}$, Neslihan Üstündağ Okur ${ }^{4}{ }^{\oplus}$, Yağmur Özhan ${ }^{5}$, \\ Hande Sipahi ${ }^{5}\left(\right.$ ) , Sule Ayla ${ }^{6}$, Benay Daylan ${ }^{6}$, Betül Demirci ${ }^{7}$ and Fatih Demirci $7,8, *(\mathbb{D}$ \\ 1 Department of Pharmacology, Faculty of Pharmacy, University of Health Sciences, 34668 Istanbul, Turkey \\ 2 Department of Pharmacognosy, School of Pharmacy, Istanbul Medipol University, 34810 Istanbul, Turkey; \\ ayseesraguler@gmail.com \\ 3 Department of Pharmacognosy, Graduate School of Health Sciences, Anadolu University, \\ 26470 Eskişehir, Turkey \\ 4 Department of Pharmaceutical Technology, Faculty of Pharmacy, University of Health Sciences, \\ 34668 Istanbul, Turkey; neslihanustundag@yahoo.com \\ 5 Department of Pharmaceutical Toxicology, Faculty of Pharmacy, Yeditepe University, 34775 Istanbul, Turkey; \\ ozhanyagmur@hotmail.com (Y.Ö.); handesipahi@hotmail.com (H.S.) \\ 6 Department of Histology and Embryology, School of Medicine, Istanbul Medipol University, \\ 34810 Istanbul, Turkey; sayla@medipol.edu.tr (Ş.A.); benaydaylan94@gmail.com (B.D.) \\ 7 Faculty of Pharmacy, Anadolu University, 26470 Eskişehir, Turkey; betuldemirci@gmail.com \\ 8 Department of Pharmacognosy, Faculty of Pharmacy, Eastern Mediterranean University, Famagusta, \\ 99628 Famagusta, Cyprus \\ * Correspondence: evrenokurecz@gmail.com (M.E.O.); demircif@gmail.com (F.D.); \\ Tel.: +90-2164189616 (M.E.O.); +90-2223350580 (F.D.)
}

Academic Editors: Marcello Locatelli, Simone Carradori and Andrei Mocan Received: 17 March 2020; Accepted: 7 May 2020; Published: 10 June 2020

\begin{abstract}
The air-dried aerial parts of Phlomis russeliana (Sims) Lag. Ex Benth. was extracted by methanol and fractionated by $n$-hexane, dichloromethane, and ethyl acetate, respectively. The wound healing properties of $P$. russeliana extract gel was evaluated using the in vivo excisional wound model using Balb-c mice. tInitially, the P. russeliana methanol extract showed LOX inhibitory activity at $\mathrm{IC}_{50}=23.2 \mu \mathrm{g} / \mathrm{mL}$, whereas the $\mathrm{DPPH}^{\bullet}$ assay showed $\mathrm{IC}_{50}=0.89 \mathrm{mg} / \mathrm{mL}$, and the $\mathrm{ABTS}^{\bullet}$ assay showed $\mathrm{IC}_{50}=0.99 \mathrm{mg} / \mathrm{mL}$, respectively. In addition, a remarkable anti-inflammatory activity was observed in the cell culture assay. Thereafter, activity-guided fractionation was performed by LOX enzyme inhibition assays, and the structures of the two most active fractions were revealed by both GC-FID and GC/MS analyses, simultaneously. Phytol and 1-heptadecanoic acid were characterized as the active constituents. Moreover, the P. russeliana extract gel formulation was applied for in vivo tests, where the new gel formulation supported the in vitro anti-inflammatory activity findings. As a conclusion, this experimental results support the wound healing evidence based on the ethnobotanical application of Phlomis species with further potential.
\end{abstract}

Keywords: P. russeliana; bioactivity-guided fractionation; wound healing; anti-inflammatory; phytochemical analyses; mice

\section{Introduction}

Phlomis L. species of the Lamiaceae usually have aromatic characteristics, while their aerial parts have a distinctive taste. The Turkish Phlomis species are used in traditional medicine mainly as tonic, appetizer, carminative, and for wound healing properties also in Turkey [1,2]. Furthermore, there are 
immunosuppressive, anti-inflammatory, antimicrobial, and free radical scavenging activities of Phlomis species among others are reported [2,3].

P. russeliana essential oils comprise mainly sesquiterpenes and aliphatic compounds such as germacrene D, $\beta$-caryophyllene, hexadecenoic acid, and pentacosane [4]. The activity, chemistry, and ethnobotanical uses of this species were both compiled in a review [2]. The phytochemical profile of $P$. russeliana was analyzed by HPLC-PAD-MS and forsythoside B, verbascoside, samioside, isoverbascoside, leucosceptoside A, and leucosceptoside B were detected from aerial parts of this plant [5]. Moderate antimicrobial and anticancer activity of $P$. russeliana methanol extract were reported by Alpay and co-workers [6]. The bioactivity, chemistry and ethnobotanical use of this species was received extensively [2].

Wounds represent a major global health challenge, which put much economic, financial, and social stress on health institutions, health professionals, patients, and their families [7]. For centuries, plants have been used in tradition to treat and prevent diseases. Many plant preparations were used for wound repairing such as Aloe vera [8], Marrubium vulgare L. leaves [9], and Elaeis guineensis Jacq [10], among others. Wound healing is a natural body response when the skin is damaged by four programmed phases which are namely hemostasis, inflammation, proliferation, and remodeling [11]. Wound healing is a complex process since many factors are attributed. Wounds are easily colonized by bacteria or fungi hindering healing. Thus, clinicians recommend the use of topical antimicrobials in order to control the potential infection of deeper body tissues and blood circulation, which can cause sepsis [12]. Nonetheless, some topical antibiotics may show cytotoxicity delaying wound healing. Furthermore, antibiotic resistance may also complicate wound disinfection and healing. Hence, the use of medicinal plant preparations can be beneficial since they have biocompability, wound healing, and antimicrobial properties.

The purpose of this present study was to evaluate and determine the pharmacological activities of the herbal preparations of $P$. russeliana, which is known to be used in Anatolian folk medicine in wounds. For the identification of the active fraction(s), the air-dried aerial part of the plant material was extracted by methanol and fractionated by $n$-hexane, dichloromethane, and ethyl acetate, respectively. The phenolic constituents were characterized by the Folin-Ciocalteu method; antioxidant activity was performed by $\mathrm{ABTS}^{\bullet}$ and $\mathrm{DPPH}^{\bullet}$ scavenging assays, respectively. In vitro anti-inflammatory activity was evaluated both by LOX enzyme inhibition, and cell culture assays. For confirmation, the wound healing properties of $P$. russeliana extract gel formulation was studied by in vivo animal tests. To the best of our knowledge, this is the first study on the in vitro and in vivo biological activities, especially on the wound healing properties of the P. russeliana extract rooting to Turkish ethnobotanical uses.

\section{Results}

\subsection{Antioxidant Activity and Total Phenolic Content}

In the present study, the antioxidant activity of the P. russeliana methanol extract was analyzed by $\mathrm{DPPH}^{\bullet}$ and $\mathrm{ABTS}^{\bullet}$ free radical assays. A calibration curve was drawn with the results obtained in decreasing concentrations $(0.5 \mathrm{mg} / \mathrm{mL}$ and $0.2 \mathrm{mg} / \mathrm{mL})$ starting from $1 \mathrm{mg} / \mathrm{mL}$, and the respective IC50 values were calculated thereof. The $P$. russeliana methanol extract showed relatively less antioxidant activity against $\mathrm{DPPH}^{\bullet}\left(\mathrm{IC}_{50}=0.91 \mathrm{mg} / \mathrm{mL}\right)$ and $\mathrm{ABTS}\left(\mathrm{IC}_{50}=0.98 \mathrm{mg} / \mathrm{mL}\right)$ radicals compared to the standard ascorbic acid $(0.002 \mathrm{mg} / \mathrm{mL})$ and Trolox $(0.015 \mathrm{mg} / \mathrm{mL})$, respectively. Furthermore, the P. russeliana extract showed $3996 \mathrm{mg}$ of GA/100 g extract corresponding to the total phenolic amounts, resulting in relatively moderate antioxidant activity on the in vitro ABTS and $\mathrm{DPPH}^{\bullet}$ evaluations. The use of scavengers to the injured wound site was reported as effective in inflammatory conditions and wound healing [13]. The effects were attributed to the antioxidant potential, which is related to the development of matured collagen fibers and fibroblasts supporting enhanced angiogenesis [14]. Previous studies showed the antioxidant potential of $P$. russeliana extracts using the DPPH radical assay supporting our findings [6,15]. 


\subsection{Antimicrobial Activity}

In this present work, the antimicrobial activity of P. russeliana methanol extract was initially tested against human pathogenic microorganisms such as Pseudomonas aeruginosa (ATCC 10145), Escherichia coli (NRLL B-3008), Staphylococcus aureus (ATCC 6538), Helicobacter pylori (ATCC 43504), Staphylococcus epidermidis (ATCC 14990), and Candida albicans (ATCC 90028). Table 1 shows the antimicrobial activity of $P$. russeliana $\mathrm{MeOH}$ extract and the standards versus the tested pathogenic microorganisms. The $P$. russeliana $\mathrm{MeOH}$ extract showed no remarkable antimicrobial activity against the tested human pathogenic microorganisms at the tested $(10 \mathrm{mg} / \mathrm{mL})$ concentrations except $H$. pylori. However, a minimum inhibitory concentrations (MIC) value of $P$. russeliana methanol extract of $5 \mathrm{mg} / \mathrm{mL}$ against $H$. pylori was determined (Table 1). Among the analyzed bacteria, $H$. pylori was relatively more sensitive to the extract, given that the others appeared to be resistant. Phlomis species are rich in essential oil content. As already reported in previous studies $[2,4,6,16]$, the apolar fractions and essential oils of the Phlomis species are also remarkable for their antimicrobial activity. In a study conducted by our group with $P$. russeliana essential oil, the effectiveness of this essential oil against Bacillus cereus and Aeromonas hydrophila was observed [4]. In another previous study on P. russeliana polar extracts, antimicrobial effects against the pathogens S. aureus, Proteus vulgaris, Salmonella typhi, Kluyveromyces fragilis, Rhodotorula rubra, Debaryomyces hansenii, Staphylococcus pyogenes, and Klebsiella pneumonia were reported [6,16].

Table 1. Antimicrobial activity of P. russeliana methanol extract (MICs in $\mathrm{mg} / \mathrm{mL}$ ).

\begin{tabular}{ccccccc}
\hline Samples & E. coli & S. aureus & S. epidermidis & P. aeruginosa & H. pylori & C.albicans \\
\hline MeOH extract & $>10$ & $>10$ & $>10$ & 10 & 5 & 10 \\
\hline Amoxicillin & 0.5 & $\leq 0.125$ & 4 & $>16$ & $\leq 0.125$ & $\mathrm{nt}$ \\
Clarithromycin & 0.5 & 0.25 & $\leq 0.125$ & $>16$ & 0.025 & $\mathrm{nt}$ \\
Tetracycline & 16 & 0.25 & $>16$ & $>16$ & 0.025 & $\mathrm{nt}$ \\
Ketoconazole & $\mathrm{nt}$ & $\mathrm{nt}$ & $\mathrm{nt}$ & $\mathrm{nt}$ & $\mathrm{nt}$ & 0.25 \\
\hline
\end{tabular}

\subsection{LOX Enzyme Inhibition Assay}

P. russeliana methanol extract was investigated for its biological evaluation by LOX inhibition assay. The tested extract showed moderate LOX inhibitory activity $\left(\mathrm{IC}_{50}=23.2 \pm 3.9 \mu \mathrm{g} / \mathrm{mL}\right)$ when compared to standard NDGA ( $\mathrm{IC}_{50}=3.2 \pm 0.9 \mu \mathrm{g} / \mathrm{mL}$, Table 2$)$. Additionally, these obtained results were supported by the in vitro cell culture assays. Bioactivity-guided fractionation was performed over LOX enzyme inhibition. Sub-extracts and fractions were also evaluated for LOX enzyme inhibition. The $\mathrm{IC}_{50}$ value of $n$-hexane extract was calculated as $11.8 \pm 1.9 \mu \mathrm{g} / \mathrm{mL}$, and the $\mathrm{IC}_{50}$ values of dichloromethane and ethyl acetate extracts were calculated as $17.6 \pm 2.1$ and $23.9 \pm 2.8 \mu \mathrm{g} / \mathrm{mL}$, respectively. Nine different fractions (Pr-H1 to Pr-H9) from $n$-hexane extract were also evaluated for LOX enzyme inhibition and LOX enzyme inhibition was found highest in the bioactive Pr-H6 fraction $(9.9 \pm 0.9 \mu \mathrm{g} / \mathrm{mL}$, Table 2). Seven different sub-fractions (Pr-H6.1 to Pr-H6.7) from Pr-H6 were also evaluated for LOX enzyme inhibition, and significantly significant LOX enzyme inhibition was observed in two of these seven fractions (Pr-H6.3 $(4.1 \pm 1.1 \mu \mathrm{g} / \mathrm{mL})$ and Pr-H6.4 $(4.9 \pm 1.3 \mu \mathrm{g} / \mathrm{mL}))$. As already well known, lipoxygenases are the key players in the formation of various physiologically active compounds of the leukotriene, lipoxin, and oxylipin families, by regulating cell metabolism and apoptosis. Moreover, these enzymes are among the most important targets for inhibition, since have been reported to play a role also in the wound healing process. Several studies recently revealed that the absence of 5-lipoxygenase leads to faster skin wound closure $[17,18]$. 
Table 2. LOX enzyme inhibition of P. russeliana extracts and fractions (in $\mathrm{g} / \mathrm{mL}$ ).

\begin{tabular}{cc}
\hline Samples & IC $_{\mathbf{5 0}}$ \\
\hline MeOH extract & $23.2 \pm 3.9$ \\
n-hexane sub-extract & $11.8 \pm 1.9$ \\
dichloromethane sub-extract & $17.6 \pm 2.1$ \\
Ethyl acetate sub-extract & $23.9 \pm 2.8$ \\
Pr-H1 & $43.9 \pm 3.9$ \\
Pr-H2 & $29.7 \pm 2.4$ \\
Pr-H3 & $19.3 \pm 1.7$ \\
Pr-H4 & $23.4 \pm 3.1$ \\
Pr-H5 & $14.1 \pm 2.3$ \\
Pr-H6 & $9.9 \pm 0.9$ \\
Pr-H7 & $11.7 \pm 1.8$ \\
Pr-H8 & $13.9 \pm 3.4$ \\
Pr-H9 & $16.5 \pm 1.5$ \\
Pr-H6.1 & $18.9 \pm 2.8$ \\
Pr-H6.2 & $17.4 \pm 1.6$ \\
Pr-H6.3 & $4.1 \pm 1.1$ \\
Pr-H6.4 & $4.9 \pm 1.3$ \\
Pr-H6.5 & $13.4 \pm 3.1$ \\
Pr-H6.6 & $39.1 \pm 2.3$ \\
\hline NDGA & $3.2 \pm 0.9$ \\
\hline
\end{tabular}

\subsection{Bioactivity-Guided Fractionation and Chemical Characterization}

After sub-extracts were prepared with $n$-hexane, dichloromethane, and ethyl acetate, respectively, fractionation was performed by open column chromatography over bioactive $n$-hexane sub-extract. Pr-H1, Pr-H2, Pr-H3, Pr-H4, Pr-H5, Pr-H6, Pr-H7, Pr-H8, and Pr-H9 were obtained after column chromatography. After all the fractions were evaluated for LOX enzyme inhibition, fractionation was continued on the bioactive fraction, Pr-H6, and two highly active subfractions (Pr-H6.3 and Pr-H6.4) were obtained from Pr-H6 (Scheme S1, in Supplementary Files).

The $P$. russeliana active fractions, which were obtained from $n$-hexane sub-extract, were analyzed by using GC-FID and GC-MS to determine the bioactive constituents, which were identified as 1-heptadecanol (10.3-20.4\%) and phytol (34.5-78.3\%). According to previous work on P. russeliana essential oils, mainly sesquiterpenoids such as $\beta$-caryophyllene, and germacrene $D$ were characterized as main constituents [4]. Also, diterpenes are an important group of compounds with anti-inflammatory activities $[19,20]$. Phytol is an acyclic diterpene alcohol, commonly found in the composition of various oils [21-23], which was found among the bioactive metabolites of $P$. russeliana $n$-hexane sub-extract, associated with anti-inflammatory activity [24]. Thus, in the present study, the remarkable anti-inflammatory activity of the bioactive fraction may be influenced from the phytol content.

\subsection{Wound Healing, Analgesic and Anti-Inflammatory Activity by Cell Culture Method}

\subsubsection{Cell Culture}

The biocompatibility of the P. russeliana methanol extract was evaluated on RAW 264.7 cell lines and L929 fibroblast on cells by MTT test after treatment with various concentrations of $P$. russeliana extract for $24 \mathrm{~h}$. As it is shown in Figure 1, P. russeliana extracts did not exhibit a cytotoxic effect on the tested cells at $0.125-1 \mathrm{mg} / \mathrm{mL}$ concentrations. In addition, a significant dose-dependent increase in RAW 264.7 cell proliferation for $0.125-0.5 \mathrm{mg} / \mathrm{mL}$ of the extract as shown in Figure 1B was observed. 
A

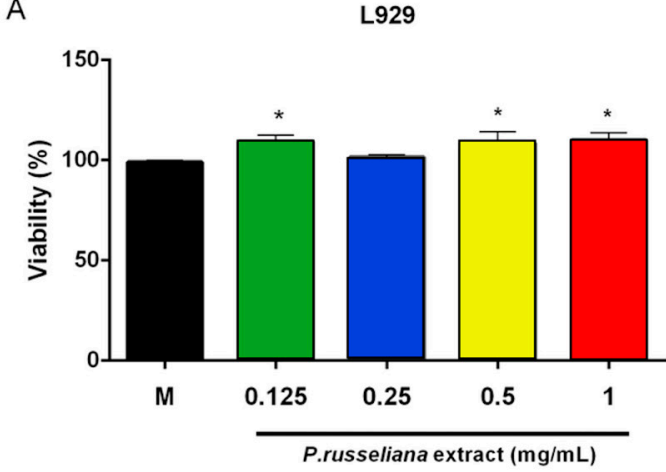

B

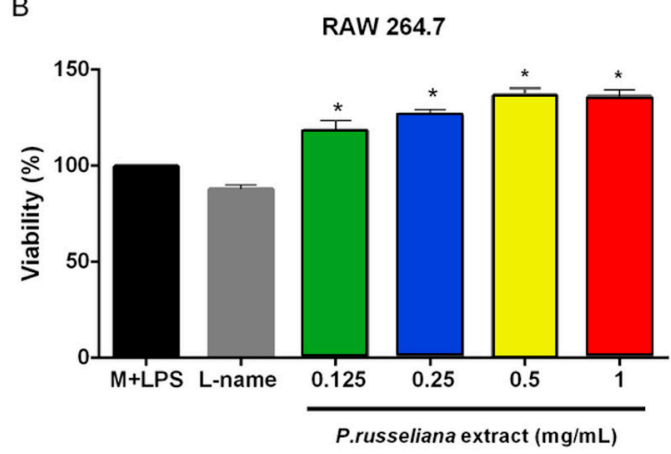

Figure 1. Effects of P. russeliana extract studied at different concentrations on L929 cell viability (A) and RAW264.7 cell viability (B). L-name: $N-(\gamma)$-nitro- $L$-arginine methyl ester $\left.{ }^{*} p<0.05\right)$.

\subsubsection{Analgesic and Anti-Inflammatory Activity}

The inflammation phase on the wound healing process plays a major role in activating the inflammatory cytokines and chemokines and in recruiting macrophages which could improve the wound healing rate [25]. However, inflammation, which might occur, also damages nearby tissues. Consequently, anti-inflammation is beneficial for wound treatment.

Furthermore, macrophages react in inflammatory responses through the generation of various cytokines. The activated macrophages act regarding pathogen invasion via the release of various pro-inflammatory cytokines and inflammatory mediators such as nitric oxide [26]. The P. russeliana extract was tested at $0.125-1 \mathrm{mg} / \mathrm{mL}$ concentrations for their inhibitory activities against lipopolysaccharide (LPS)-induced nitrite production in RAW 264.7 cells. The extract exerted anti-inflammatory activity dose-dependent fashion. The comparison of anti-inflammatory activity of the extract with the reference substance NOS inhibitor L-name $(0.1 \mathrm{mg} / \mathrm{mL})$, is shown in Figure 2.

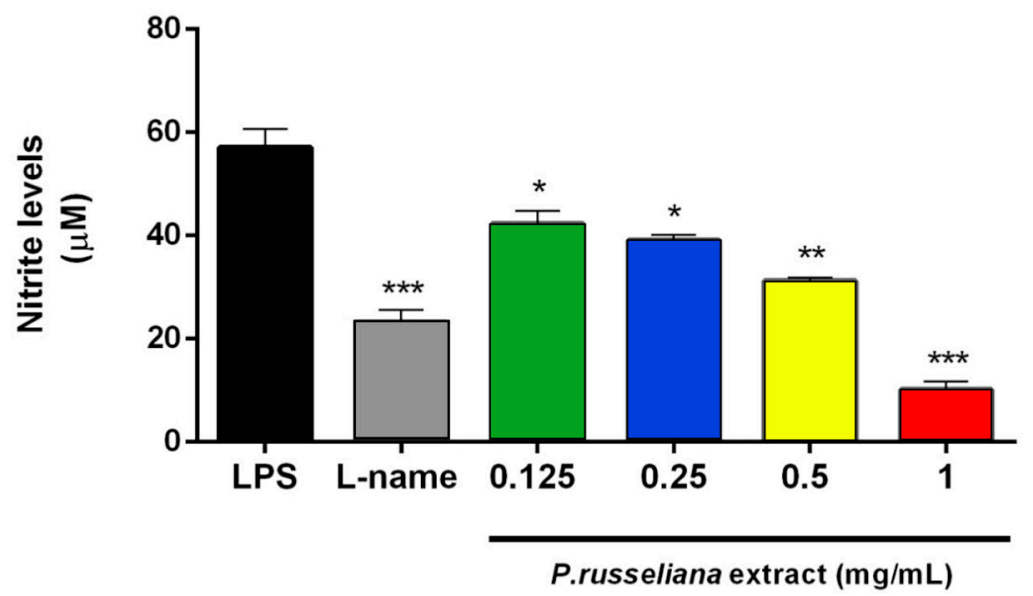

Figure 2. Effects of P. russeliana extract on nitrite production in RAW264.7 cells stimulated with $1 \mu \mathrm{g} / \mathrm{mL}$ of LPS. LPS: Control group treated with $1 \mu \mathrm{g} / \mathrm{mL}$ LPS. Statistical significances were indicated for each compound vs. LPS $\left.{ }^{*} p<0.05,{ }^{* *} p<0.01,{ }^{* * *} p<0.001\right)$.

The results revealed that the extract may work as an efficient anti-inflammatory agent by inhibiting the production of NO. The inhibition of NO production in macrophages could possibly be due to the presence of phenols, since various studies demonstrated that phenols can act as anti-inflammatory agents, and they play a key role in oxidative stress and inflammation [26].

Pain is a highly unpleasant physical sensational and emotional experience associated with actual or potential tissue damage. The unresolved wound pain can have a negative impact on wound healing by delaying the process [27]. 
In this present work, the possible analgesic potency of the P. russeliana extract is remerkable since it can serve both as a wound healing and analgesic agent. It is well established that the release of high levels of prostaglandins $\left(\mathrm{PGE}_{2}\right)$ is produced in response to injury or infection, and may causes inflammation, which is associated with the symptoms of redness, swelling, pain, and fever [28]. Thus, herein, the analgesic activity on $\mathrm{PGE}_{2}$ productions was evaluated for the extract doses with anti-inflammatory activity. As in Figure 3, both P. russeliana extract $(0.25-1 \mathrm{mg} / \mathrm{mL})$ and N(gamma)-nitro-L-arginine methyl ester (L-NAME; $0.1 \mathrm{mg} / \mathrm{mL}$ ) inhibited LPS-induced production of $\mathrm{PGE}_{2}$. Therefore, reduction/termination of the persistent inflammation and elimination of free radicals by the introduction of an antioxidant could be an important strategy to improve healing [29].

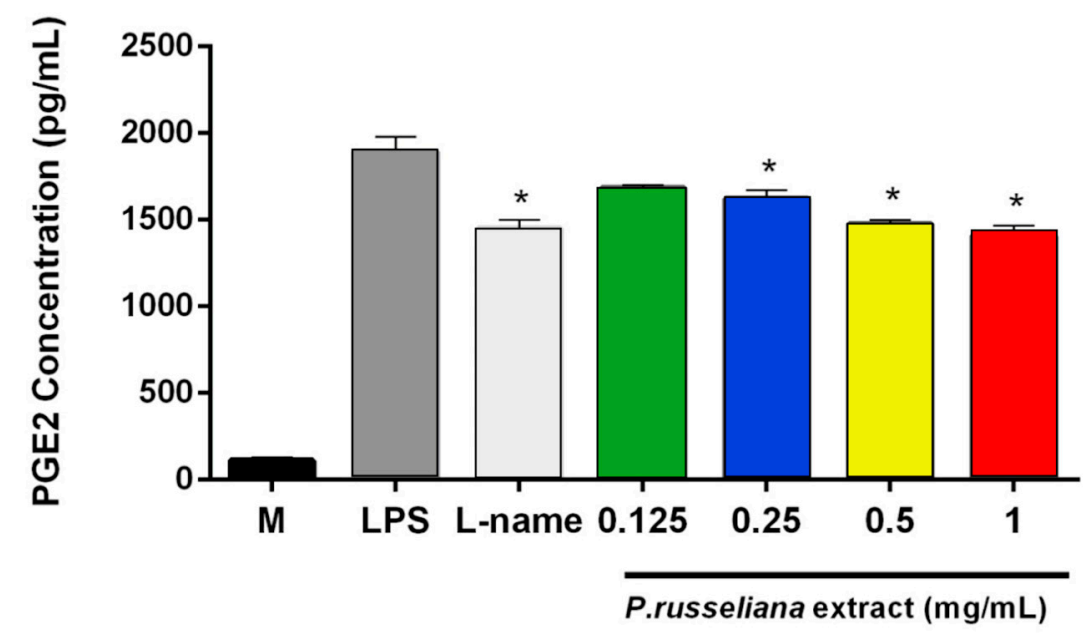

Figure 3. Analgesic effect of P. russeliana extract on LPS-induced production of $\mathrm{PGE}_{2}$. LPS: Control group treated with $1 \mu \mathrm{g} / \mathrm{mL}$ LPS. L-name: $N-(\gamma)$-nitro- $L$-arginine methyl ester. Statistical significances were indicated for each compound vs. LPS $\left({ }^{*} p<0.05\right)$.

\subsection{In Vivo Wound Healing}

As it is well known, the wound healing process comprises three different phases. The first phase is when the inflammation occurs; consequently, the neutrophils and macrophages accumulate to the lesion location. The following stage is the proliferation, when re-epithelization, angiogenesis, fibroplasia, and granulation tissue formation happen. Finally, in the remodeling phase, the blood vessels amount (neovascularization) is reduced and the neocollagenesis course occurs. Subsequently, the wound is diminished. The target of wound healing is either to reduce the healing time or to avail undesired complications such as scarring [30].

Figures 4 and 5 depict the macroscopic healing rates of wounds on the studied animals, the wound surface size of the studied group was monitored on days 0,5 , and 10 , respectively. The control, blank gel and the control Madecassol ${ }^{\circledR}$ showed wound scabs on day 5. on day 10, P. russeliana extract gel and Madecassol ${ }^{\circledR}$ depicted healing since a characteristic lesion can be observed.

In Figure 5, the percentage of wound healing rates can be observed comparatively. More specifically, on Day 5, P. russeliana gel and Madecassol ${ }^{\circledR}$ showed similar wound area percentages, which were relatively more, compared to control and blank gel. In addition, on Day 10 significant reductions in the wound surface were observed for for the P. russeliana gel and Madecassol ${ }^{\circledR}$ since both wounds were almost recovered. 


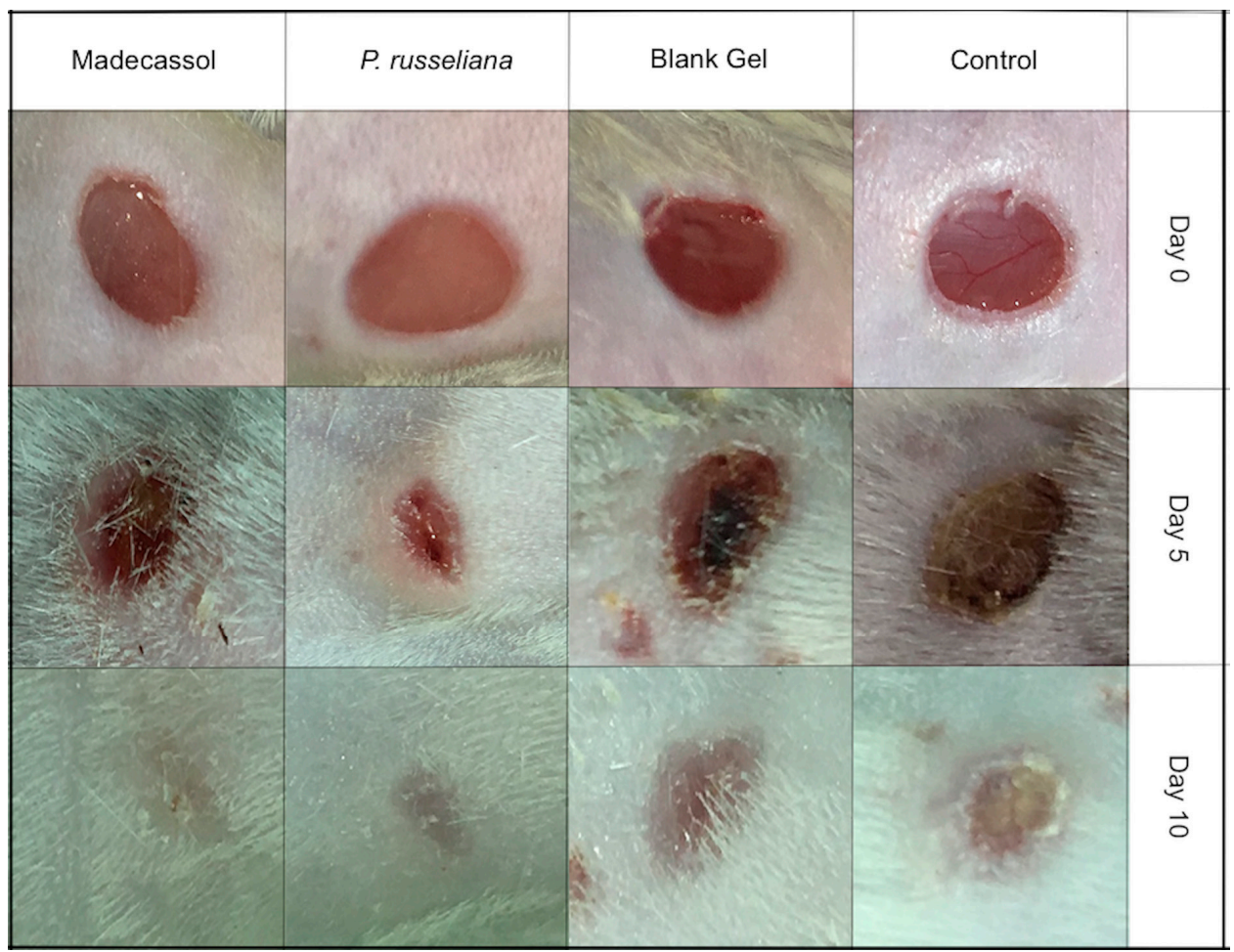

Figure 4. Macroscopic visualization of test samples: control, blank gel, Madecassol, P. russeliana extract test groups at days $0 ., 5$. and 10 .

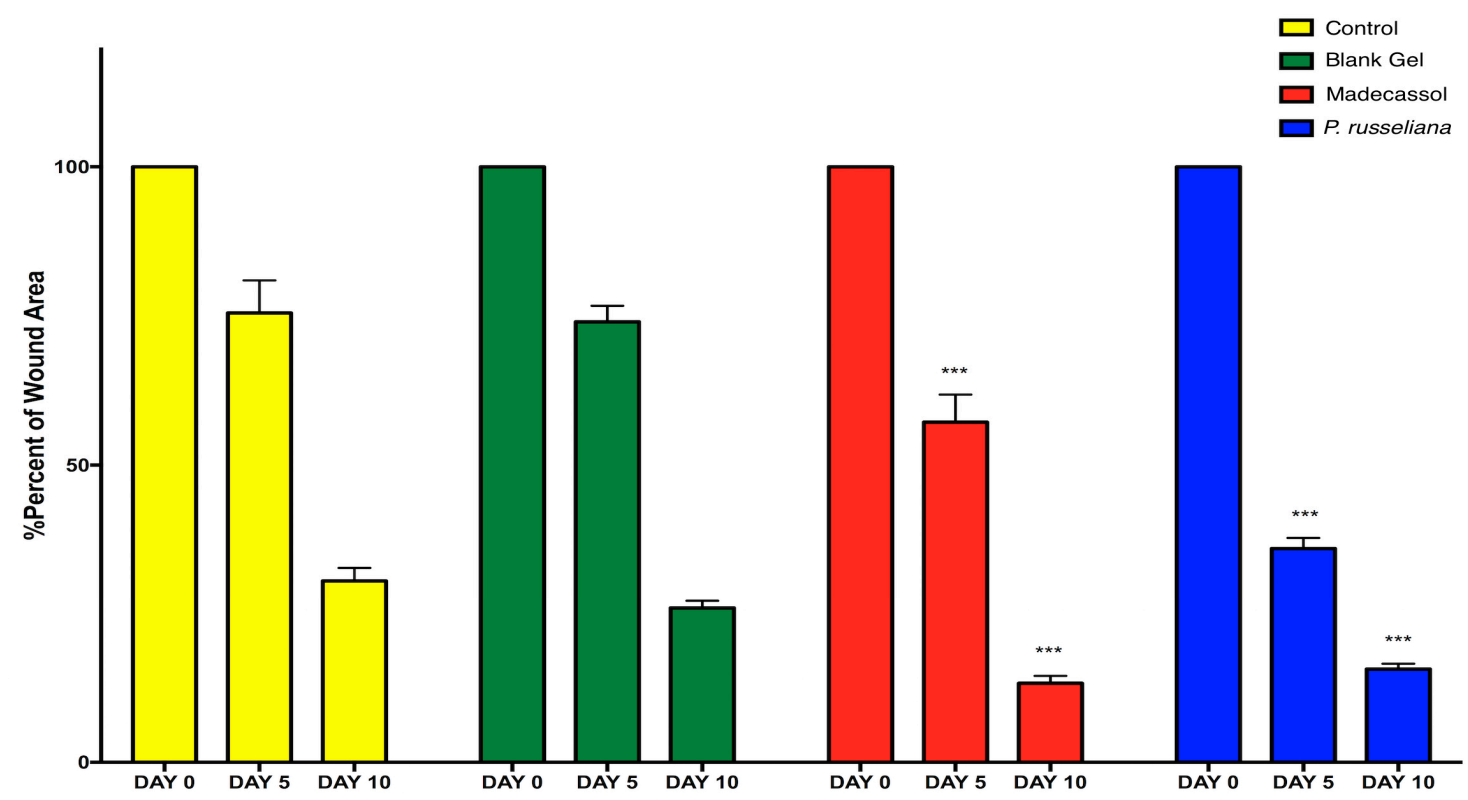

Figure 5. Healing percentages of the wound area in each test group (control, blank gel, Madecassol, P. russeliana extract gel). Statistically significant, compared to control groups; ${ }^{* * *} p<0.001$.

\subsection{Histological Evaluation of Wound Healing}

The proliferative phase presents various stages, such as angiogenesis, epithelialization, the formation of granulation tissue, fluid loss protection as well as bacterial invasion. In general, angiogenesis impacts several processes including wound healing, embryonic development, tumor growth, and chronic inflammation. Herein, angiogenesis, granulation tissue thickness, and epidermal-dermal regeneration, TGF-b, vascular endothelial growth factor (VEGF), FGF, and collagen were examined separately. 
The histopathological observation of the wound area by hematoxylin and eosin (H\&E) and immunehistochemical staining is shown in Figure 6.

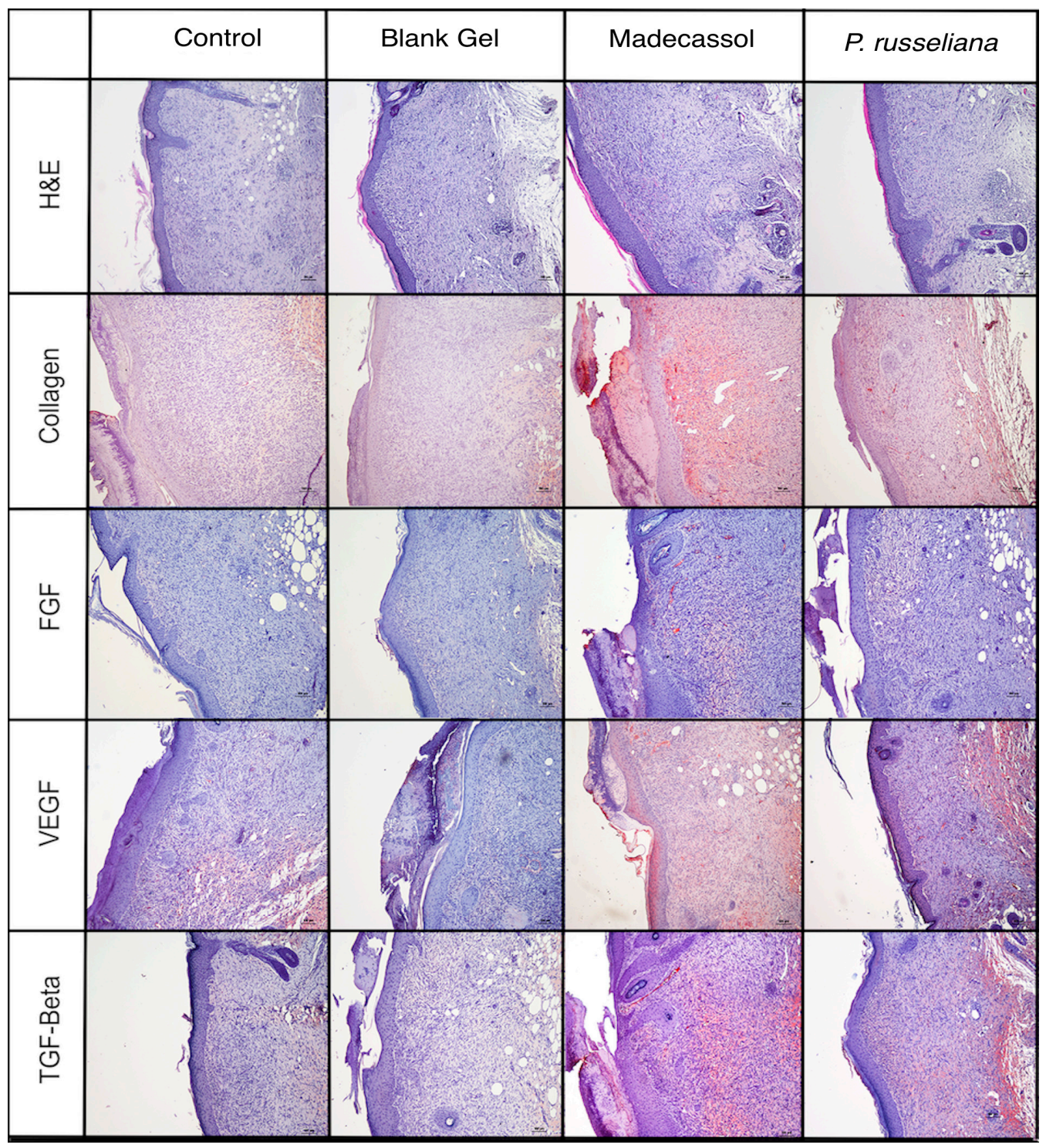

Figure 6. Histopathological view of injured tissues of the untreated (control), blank gel, extract gel, and Madecassol groups on the 10th day after wound incision (magnification 10×).

Figure 7 showed both that Madecassol and P. russeliana extract gel significantly $(p<0.001)$ affect angiogenesis, epidermal, and dermal granulation as well as granulation tissue thickness compared to control and blank gel. In addition, the vast formation of blood vessels was depicted again both for Madecassol and P. russeliana extract gel $(p<0.001)$ in comparison results suggest that the group treated with $P$. russeliana extract gel demonstrate new blood vessel formation similarly to Madecassol treated group.

Besides angiogenesis, the improvement of dermal and epidermal regeneration, which consists of well-structured epithelial layers with an absence of crusting or intraepithelial inflammatory cells, is essential for wound healing. Herein, great dermal and epidermal regeneration was revealed after the treatment of the groups with Madecassol ${ }^{\circledR}$ and P. russeliana gels compared to control and blank gel (Figure 7). It can be argued that the P. russeliana gel is similarly effective as Madecassol ${ }^{\circledR}$ gel on epithelialization, granulation tissue thickness, and angiogenesis significant for the evaluation of wound 
healing. Moreover, the observed in vivo wound healing can be associated with the presence of phytol, which can accelerate and enhance the wound healing [30].

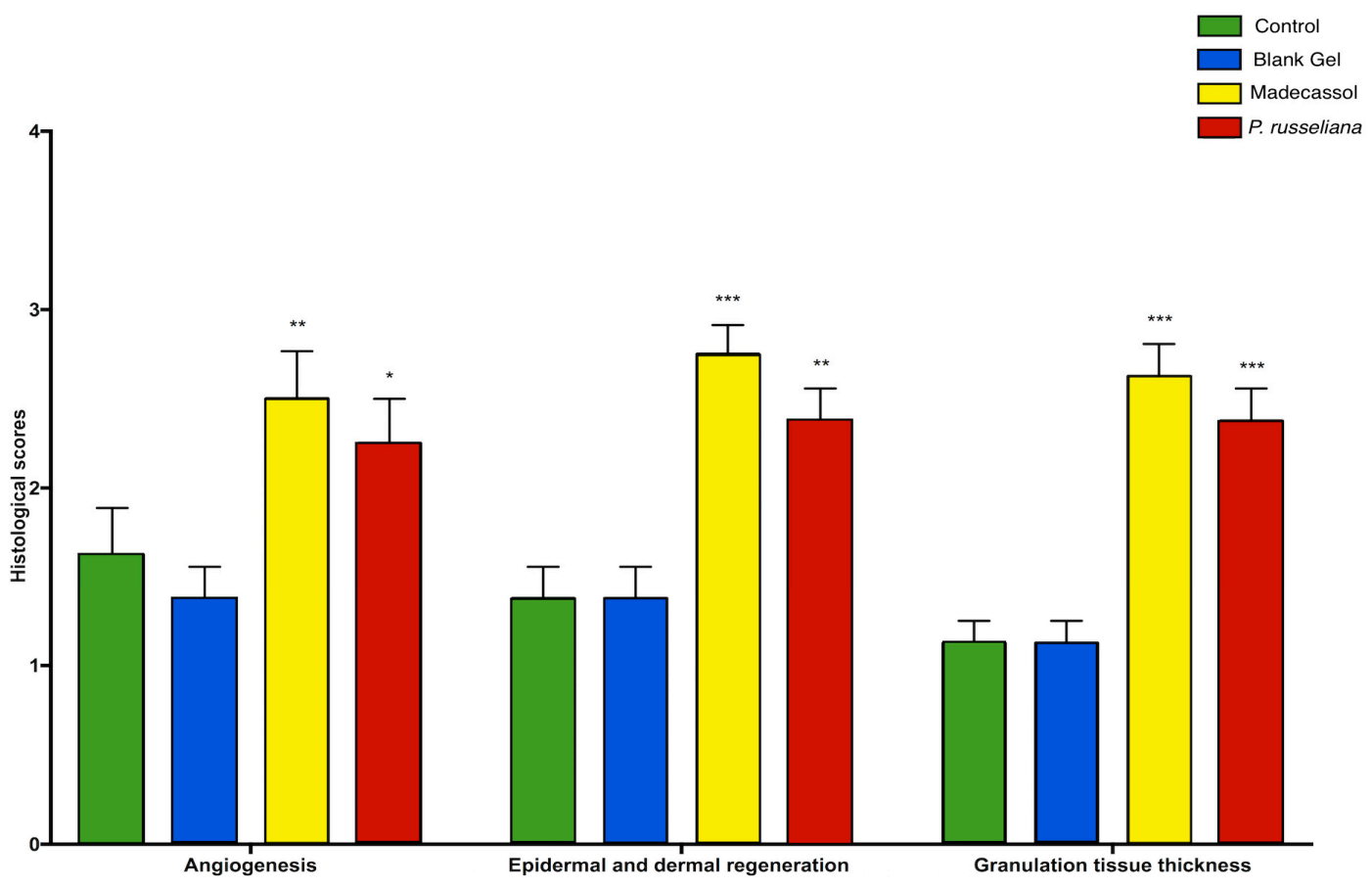

Figure 7. Histological granulation scores; tissue thickness, angiogenesis, and epidermal-dermal regeneration of control, blank gel, Madecassol, P. russeliana extract gel groups. Statistically significant compared to control groups; ${ }^{*} p<0.05,{ }^{* *} p<0.01,{ }^{* * *} p<0.001$.

Figure 8 depicts the comparison of collagen formation scores per group. Collagen is structurally and functionally a key protein of the extracellular matrix which is also involved in scar formation during the healing of connective tissues. Collagen deposition and can supply the strength and integrity that wounds need and can provoke re-epithelization. The strength of the recovered wound tissue is a result of the remodeling of collagen and the formation of stable intra and intermolecular cross-linking to form fiber [31]. Herein, both Madecassol $(p<0.001)$ and P. russeliana gel $(p<0.001)$ treated groups demonstrated greater statistically collagen formation (over two-fold collagen formation) compared to blank and control groups.

Figure 8 also exhibited the comparison of immunohistochemistry scores for TGF-b. The TGF-b, platelet-derived growth factor (PDGF), fibroblast growth factor (FGF), epidermal growth factor (EGF) as well as vascular endothelial growth factor (VEGF) are found in reduced levels on chronic wounds. In further, chronic wounds show decreased levels of interleukins 1 and 6 (IL-1 and -6) and tumor necrosis factor-a (TNF-a) [32]. In addition, TGF-b has shown potent characteristics as wound healing growth factor and can enhance wound healing rate and avoidance of scarring. In this study, it was shown (Figure 8 that the levels of TGF-b in Madecassol $(p<0.001)$ and P. russeliana gel groups $(p<0.001)$ were significantly greater than control and blank gel groups.

Moreover, Figure 8 demonstrated the VEGF scores of the studied groups. VEGF is quite significant since it is involved in granulation tissue formation and thus further enhanced angiogenesis and wound healing [33]. It revealed that Madecassol $(p<0.001)$ and P. russeliana gels $(p<0.01)$ had a significant increase of VEGF scores (more than two-fold increase) in comparison with the control group and blank gel groups as observed in Figure 8. 


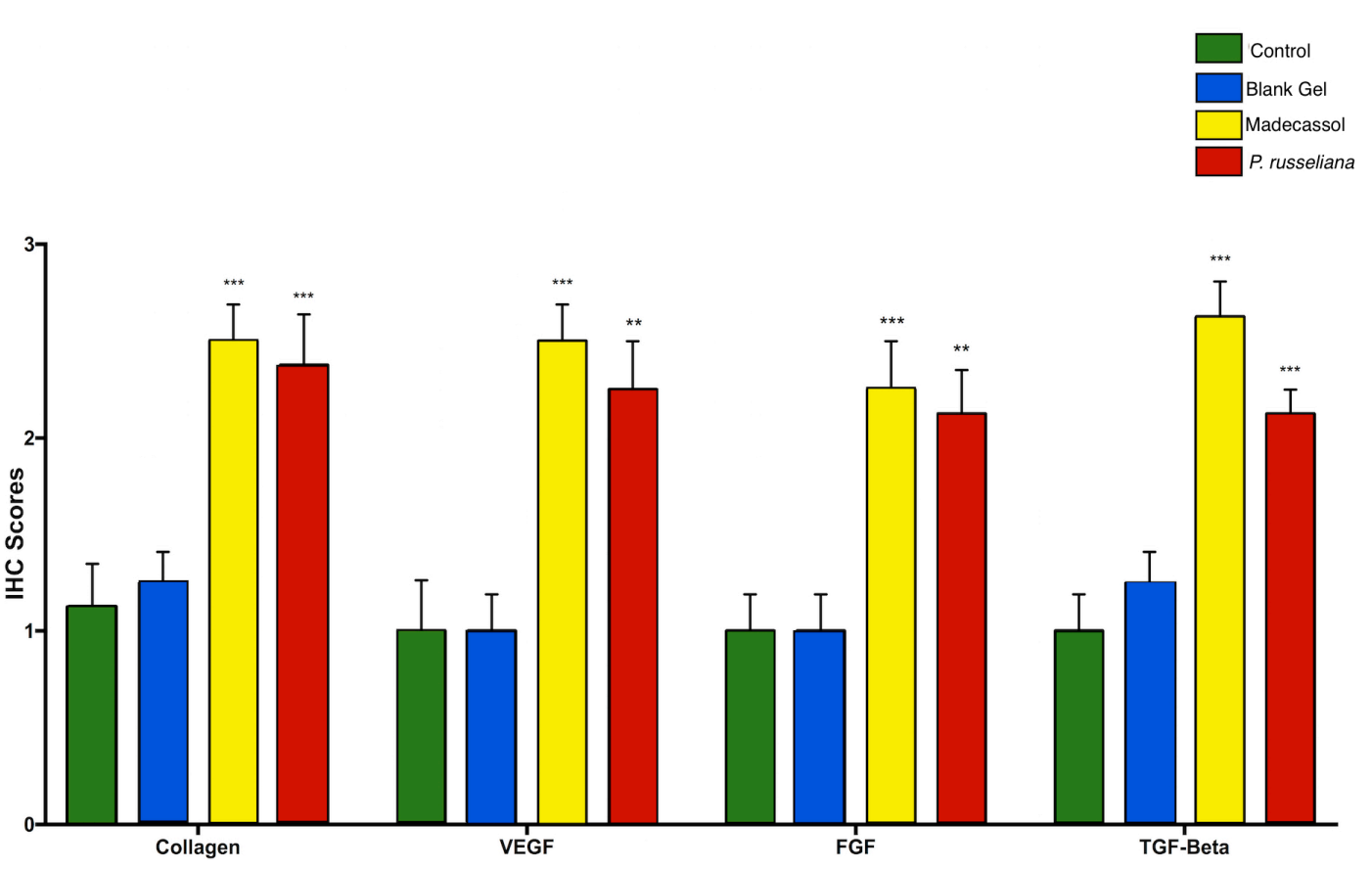

Figure 8. Comparison of immunohistochemistry; collagen, TGF-Beta, VEGf, FGF wound healing scores among the test groups. Statistically significant compared to control groups; ${ }^{* *} p<0.01,{ }^{* * *} p<0.001$.

The comparison of immunohistochemistry scores for FGF, resulted in the multi-potentiality of basic FGF, which plays an important role at the site of injury and is known to promote wound healing, as also confirmed in Figure 8. FGF was reported to enhance the proliferation of human periodontal ligament cells in the oral area [34]. In this study, it was shown that the expression of FGF in Madecassol $(p<0.01)$ and P. russeliana gel groups $(p<0.001)$ was significantly greater than control and blank gel groups.

\section{Materials and Methods}

\subsection{Materials}

Carbopol and Glycerine were purchased from Sigma, Germany. Lipoxygenase (1.13.11.12, type I-B, Soybean), linoleic acid, and LPS (lipopolysaccharide from Escherichia coli 0111:B4) were purchased from Sigma (St. Louis, MO, USA). The RAW 264.7 murine macrophage cell line and L929 healthy mouse fibroblast were purchased from ATCC (Gaithersburg, MD, USA) and DMEM was purchased from Gibco (New York, NY, USA). All the other chemicals and solvents were of analytical or HPLC grade.

\subsection{Plant Material, Extraction, and Bioactivity-Guided Fractionation}

P. russeliana was collected in June 2015 from Abant, Bolu, Turkey. Voucher specimens were deposited in the Herbarium of the Faculty of Pharmacy, Istanbul University (ISTE 115022). The air-dried aerial parts were crushed and extracted with methanol by maceration followed by filtration and evaporation (Heidolph, Darmstadt, Germany). The sub-fractions were prepared via liquid-liquid extraction using $n$-hexane, dichloromethane, and ethyl acetate, respectively.

The $n$-hexane extract ( $4 \mathrm{~g}$ ) was purified using a Silica column $(400 \mathrm{~g})$ eluting with $n$-hexane and stepwise gradient of ethyl acetate in $n$-hexane to yield nine fractions, from Pr-H1 to Pr-H9. The Pr-H6 $(250 \mathrm{mg})$ was applied to a Silica $(20 \mathrm{~g})$ column and eluted with $n$-hexane and a gradient of ethyl acetate in $n$-hexane to obtain further six fractions, from Pr-H6.1 to Pr-H6.6. The phytochemistry of Pr-H6.3 and Pr-H6.4 were determined by GC-MS/GC-FID. 


\subsection{GC-FID and GC/MS Analyses}

The bioactive fractions of $P$. russeliana were analyzed by an Agilent 5975 GC-MSD system. The used column was the Innowax FSC $(60 \mathrm{~m} \times 0.25 \mathrm{~mm}, 0.25 \mu \mathrm{m}$ film thickness $)$ using helium as carrier gas $(0.8 \mathrm{~mL} / \mathrm{min})$. The $\mathrm{GC}$ oven temperature set at $60^{\circ} \mathrm{C}$ for $10 \mathrm{~min}$ and programmed to $220^{\circ} \mathrm{C}$ at a rate of $4{ }^{\circ} \mathrm{C} / \mathrm{min}$, and kept constant at $220^{\circ} \mathrm{C}$ for $10 \mathrm{~min}$ and then programmed to $240{ }^{\circ} \mathrm{C}$ at a rate of $1{ }^{\circ} \mathrm{C} / \mathrm{min}$. The split ratio was adjusted at $40: 1$. The injector temperature was set at $250^{\circ} \mathrm{C}$. The mass spectra were recorded at $70 \mathrm{eV}$ and the mass range was from $\mathrm{m} / \mathrm{z} 35$ to 450 .

The GC analysis was carried out using an Agilent 6890N GC system. The temperature of FID detector was $300^{\circ} \mathrm{C}$. A simultaneous auto-injection was done on a duplicate of the same column applying the same operational conditions in order to obtain the same elution order with GC/MS. The relative percentage amounts (\%) of the separated compounds were calculated from FID chromatograms.

The identifications of the oil components were performed by comparison to their relative retention times (RRT) with those of authentic samples or compared to their relative retention index (RRI) to series of $n$-alkanes. The computer matching of the separated compounds against commercial (Wiley GC/MS Library, MassFinder Software 4.0) and the in-house "Başer Library of Essential Oil Constituents" built up by genuine compounds and components from literature previous reported [3,4,21].

\subsection{In Vitro Biological Assays}

\subsubsection{Antioxidant Activity and Total Phenolic Content}

\section{Radical Scavenging Activity by DPPH Method}

Radical scavenging activity of the extract was detected by its capacity of bleaching the stable radical DPPH [35]. The reaction mixture comprised of $100 \mu \mathrm{M} \mathrm{DPPH}{ }^{\bullet}$ in methanol and P. russeliana $\mathrm{MeOH}$ extract. The mixture's absorbance was determined at $517 \mathrm{~nm}$ by a UV spectrophotometer (UV-1800, Shimadzu, Japan) at room temperature. The radical scavenging activity percentage inhibition (\%) was obtained as follows:

$$
\left.\%=\left[\left(\text { Absorbance }_{\text {control }^{*}}-\text { Absorbance }_{\text {test sample }}\right) / \text { Absorbance }_{\text {control }}{ }^{*}\right)\right] \times 100
$$

( ${ }^{*}$ Control; Ascorbic acid)

\section{ABTS Radical Scavenging Assay}

The antioxidant capacity of the P. russeliana $\mathrm{MeOH}$ extract was determined according to previous literature [36]. The solution was prepared by mixing potassium persulfate and ABTS ${ }^{\bullet}$ and allowing them to react at room temperature for $16 \mathrm{~h}$ in the dark. The absorbance was set at $734 \mathrm{~nm}$. The study was performed in triplicate. The percentage inhibition (\%) was calculated as:

$$
\left.\%=\left[\left(\text { Absorbance }_{\text {control }^{*}}-\text { Absorbance }_{\text {test sample }}\right) / \text { Absorbance }_{\text {control }}{ }^{*}\right)\right] \times 100
$$

$\left({ }^{*}\right.$ Control; Trolox $)$

\section{Total Phenolic Content}

Folin-Ciocalteu method was used for the determination of the total phenolics content of $P$. russeliana $\mathrm{MeOH}$ extract. Folin-Ciocalteau's reagent and $\mathrm{Na}_{2} \mathrm{CO}_{3}$ were mixed with extract and allowed to incubate at $45^{\circ} \mathrm{C}$ for $15 \mathrm{~min}$. The absorbance was determined at $765 \mathrm{~nm}$ at room temperature. The total phenolic ingredient was measured from a linear calibration curve $\left(R^{2}=0.9936\right)$ [37]. 


\subsubsection{Antimicrobial Activity}

The in vitro antimicrobial activity was evaluated by determining the minimum inhibitory concentrations (MIC) via the broth microdilution protocol according to the Clinical and Laboratory Standards Institute [38]. The commonly found microorganisms on colonized wounds were studied; S. aureus, P. aeruginosa, S. epidermidis, and E. coli strains were grown in Mueller Hinton Broth (MHB, Merck, Germany); C. albicans species were grown in RPMI broth at $37^{\circ} \mathrm{C}$ in aerobic conditions for $24 \mathrm{~h}$. Bacteria suspension was adjusted to $0.5 \mathrm{McFarland}$ turbidity standards (corresponding to $10^{8}$ colony-forming units $(\mathrm{CFU}) / \mathrm{mL}$ ) with sterile saline.

The H. pylori strain was grown in Brucella broth (Sigma Aldrich) at $37^{\circ} \mathrm{C}$ under anaerobic atmosphere for $24 \mathrm{~h}$. After incubation, $100 \mu \mathrm{L}$ of 1:10 diluted and transferred to the microplates with an inoculum of $2 \times 10^{7} \mathrm{CFU} / \mathrm{mL}$ of $\mathrm{H}$. pylori [39]. The diluted bacterial suspensions were added in each well and then incubated at $37^{\circ} \mathrm{C}$ for $24 \mathrm{~h}$.

All samples were prepared in dimethyl sulfoxide (DMSO). MIC (as $\mu \mathrm{g} / \mathrm{mL}$ ) was defined as the lowest concentration that inhibited visible growth. The MIC was screened and the results were compared to positive controls as shown in Table 1. Each experiment was replicated thrice.

\subsubsection{LOX Enzyme Inhibition Assay}

The 5-lipoxygenase assay (5-LOX inhibiting activity) was measured by modifying the spectrophotometric method [40]. Potassium phosphate buffer (1,94 mL;100 mM; pH 9.0), $40 \mu \mathrm{L}$ of test solution, and $20 \mu \mathrm{L}$ of lipoxygenase solution were mixed and incubated for $10 \mathrm{~min}$ at $25^{\circ} \mathrm{C}$. The reaction was initiated by the addition of $10 \mu \mathrm{L}$ linoleic acid solution, the change of absorbance at $234 \mathrm{~nm}$ was followed for $10 \mathrm{~min}$.

The test sample and the Nordihydroguaiaretic acid (NDGA, positive control) were dissolved in $\mathrm{MeOH}$. All the kinetic experiments were performed in quartz cuvette as triplicate. The concentration of the extracts was calculated for $\mathrm{IC}_{50}$ value. All test and control assays were corrected by blanks for non-enzymatic hydrolysis. The absorbance change per minute was determined. The percentage of inhibition was calculated as the absorbance change per minute of enzyme activity (without any inhibitor) compared to absorbance change per minute of the test sample.

\subsection{In Vitro Anti-Inflammatory, Analgesic, and Wound Healing Evaluation of P. russeliana MeOH Extract}

\subsubsection{Cytotoxicity Assay}

The RAW 264.7 and L929 healthy mouse fibroblasts were cultured in DMEM supplemented with $10 \% \mathrm{FBS}$ and $1 \%$ penicillin $(10.000$ units $/ \mathrm{mL})$ and streptomycin $(10.000 \mu \mathrm{g} / \mathrm{mL})$ at $37^{\circ} \mathrm{C}$ at a humidified atmosphere of $5 \% \mathrm{CO}_{2}$. For the analysis of the cytotoxicity, MTT colorimetric assay was used [41]. The plated RAW 264.7 cells and L929 cells were treated with various concentrations of $P$. russeliana extract $(0.125,0.250,0.5$, and $1 \mathrm{mg} / \mathrm{mL})$. After incubation for $24 \mathrm{~h}$, the medium was removed and MTT assay reagent $(0.5 \mathrm{mg} / \mathrm{mL})$ was added to wells and cells were incubated at $37^{\circ} \mathrm{C}$. After $2 \mathrm{~h}$ incubation, the cell culture medium was removed and isopropanol $(100 \mu \mathrm{L})$ was added to dissolve the formazan crystals. The plates were then read by a microplate reader (Thermo Multiscan Spectrum, Vantaa, Finland) at a wavelength of $570 \mathrm{~nm}$ spectrophotometrically. The percentage of cell viability was calculated according to the formula:

$$
\text { Viability } \%=\left[(\text { Absorbance } \text { treatment group })\left(\text { Absorbance }_{\text {control }}\right)\right] \times 100
$$

\subsubsection{Anti-Inflammatory Activity}

The anti-inflammatory activity of $P$. russeliana extract was assessed by determining the stable end product of nitric oxide (NO), Nitrite levels in the culture medium using the Griess reagent $(0.1 \%$ $\mathrm{N}$-(1-naphthyl) ethylenediamine dihydrochloride in $5 \%$ phosphoric acid and $1 \%$ sulfanilamide) [42]. The RAW 264.7 cells transferred in a 96-well plate at the density of $5 \times 10^{4}$ cells/well and incubated for 
$24 \mathrm{~h}$ at $37^{\circ} \mathrm{C}$ in $5 \% \mathrm{CO}_{2}$. The cells were pre-treated with the concentrations of $P$. russeliana extract $(0.125$, $0.25,0.5$, and $1 \mathrm{mg} / \mathrm{mL}$ ) for $2 \mathrm{~h}$. Afterwards, the cells were stimulated with $1 \mu \mathrm{g} / \mathrm{mL}$ of LPS for $22 \mathrm{~h}$. Then, the cell culture supernatant was collected. Fifty microliters of the supernatant were mixed with the equal volume of Griess reagent and the solution was incubated at room temperature for $10 \mathrm{~min}$ in the dark. The absorbance was measured at $540 \mathrm{~nm}$ with a microplate reader (Multiscan Ascent, Vantaa, Finland). The nitrite levels in samples were established using a sodium nitrite standard curve. As positive control, the N(gamma)-nitro-L-arginine methyl ester (L-Name; $0.1 \mathrm{mg} / \mathrm{mL}$ ) was used.

\subsubsection{Analgesic Activity}

The analgesic activity was analyzed for the samples with greater anti-inflammatory activity. The detection of Prostaglandin $\mathrm{E}_{2}\left(\mathrm{PGE}_{2}\right)$ levels in collected RAW 264.7 cell culture supernatants were achieved using the commercially available quantitative enzyme-linked immunosorbent assay (ELISA) kit (Abcam PGE 2 ELISA Kit, Cambridge, UK).

\subsection{Preparation of Topical Extract Gel}

The extract loaded gel was formulated by mixing Carbopol aqueous gel and hydroxypropyl cellulose gel. Firstly, $0.5 \mathrm{~g}$ Carbopol (dispersed in water), $7 \mathrm{~g}$ glycerin, and $20 \mathrm{~g}$ isopropyl alcohol were blended with slowly stirring using a mechanical stirrer, at $25^{\circ} \mathrm{C}$ and $3.5 \mathrm{~g}$ of triethanolamine in water was transferred. The mixture was further filled with water up to $100 \mathrm{~g}$ weight. Following, the blend was stirred constantly until the formation of a clear gel. In addition, a hydroxypropyl cellulose gel ( $2 \%)$ was prepared. The Carbopol gel and hydroxypropyl cellulose gel were mixed (50:50) ratio and 5\% of the extract was added following by gently stirring. Similarly, a blank gel was also prepared.

\subsection{In Vivo Experiments}

\subsubsection{Animals}

The animal experiment was performed according to the local ethics committee regulations (Istanbul Medipol University Institutional Animals Ethical Committee, Approval No: 2018-09). The European Community guidelines as accepted principles for the use of experimental animals, were adhered to.

Balb-c mice (25-28 g) were procured from Istanbul Medipol University, MEDITAM, Istanbul, Turkey. The mice were kept in regular cages with food and water ad libitum, at room temperature $\left(20 \pm 2{ }^{\circ} \mathrm{C}\right)$ with artificial light from 7.00 am to $7.00 \mathrm{pm}$.

\subsubsection{Wound Model and Experimental Groups}

Four groups of seven animals $(n=7)$ from the totally used twenty-eight mice were assigned randomly;

1. Untreated (Control) group;

2. Blank gel (Vehicle) group;

3. P. russeliana extract gel group;

4. Madecassol (Bayer, Switzerland, Standard drug) group.

Animals were anesthetized via mixture of ketamine (80-100mg/kg, intraperitoneal (i.p.)) and xylazine $(10 \mathrm{mg} / \mathrm{kg}$, i.p.). Afterwards, the back of the mice was shaved with an electric razor and a povidone-iodine solution was applied to dorsal skin. Full-thickness excisional skin wounds were created bilaterally in the dorsal skin with a 5-mm round skin biopsy punch. The gels were topically applied to the wound's surface every day for 10 days.

\subsubsection{Macroscopic Wound Healing}

The day when wounds were made was designated as Day 0 , and the process of wound healing was observed from then until Day 10 after wounding. Wound photographs were taken using a digital 
photo machine (Canon Inc., Tokyo, Japan) with an internal scale of each wound at days 0,5 , and 10 to measure wound contraction. The pictures of the wounds were documented at a $90^{\circ}$ angle to the plane of the wound. Wound surface areas were calculated using an image analyzer software (Image J.2.0 software, Bethesda, MD, USA) to assess wound healing ratios of per group.

\subsubsection{Histology and Immunohistochemistry}

Mice were sacrificed on Day 10 following the wounding and the whole wound with a margin of around $5 \mathrm{~mm}$ of ambient unwounded skin was excised for histological evaluation. All samples were fixed in $10 \%$ neutral formalin. After $24 \mathrm{~h}$, the biopsies were bisected, embedded in paraffin, and sectioned in $5 \mu \mathrm{m}$ thick layers

The $5 \mu \mathrm{m}$ thick sections were mounted on glass slides, dewaxed, rehydrated with distilled water and stained with vascular endothelial growth factor (VEGF, Santa Cruz sc-7269), fibroblast growth factor FGF (Santa Cruz sc-55520), transforming growth factor beta1 (TGF-b1, Santa Cruz sc-65378), Collagen type1 alpha1 (COL1A1, Santa Cruz sc-293182), and hematoxylin-eosin (HE) in order to be evaluated via light microscopy.

The wound was assessed according to the scoring system as previously reported [43]. Epidermal and dermal regeneration score system was: (1) Epidermal formation (poor) $\geq 20 \%$; (2) Epidermal formation (incomplete) $\geq 40 \%$; (3) Epithelial proliferation (moderate) $\geq 60 \%$; (4) Epidermal re-modeling (complete) $\geq 80 \%$. The thickness of the granulation tissue score system was; (1) thin layer; (2) moderate layer; (3) thick layer; (4) very thick layer.

The angiogenesis was evaluated by counting and identifying only mature vessels and the presence of erythrocytes in the lumen. The presence/absence of edema, thrombosis, hemorrhage, congestion, and intra/intervascular fibrin formation was evaluated to distinguish well-formed and poorly formed capillary vessels. Angiogenesis score system was: (1) High level of hemorrhage, edema, occasional congestion, and thrombosis; (2) Capillary vessels (newly formed, 3-4/site), occasional congestion, moderate edema and hemorrhage, intravascular fibrin deposition and absence of thrombosis; (3) Capillary vessels (newly formed, 5-6/site); (4) Capillary vessels (newly formed and normal-appearing $>7 /$ site).

The immune-reactivity of immune-histochemical stain was assessed via the semi-quantitative method. Five randomly selected areas were chosen for the evaluation, where the scoring system was: (0) No painting, (1) Little, (2) Medium, (3) Heavy painting [43].

\subsubsection{Statistical Analysis}

In vitro and In vivo data were expressed as mean \pm standard error of the mean (Mean \pm SEM). The statistical significance between groups was analyzed by One-way ANOVA (followed by Dunnett's post hoc test, in vivo) and Paired Samples T-Test (in vitro). The statistical analysis was carried out using GraphPad Prism 7.0 (GraphPad Software Inc., San Diego, CA, USA) and SPSS Statistics 22.0 (IBM, Chicago, IL, USA). $p<0.05$ was suggested as statistically significant.

\section{Conclusions}

The aerial parts of $P$. russeliana were extracted with various solvents and formulated by a gel preparation, which was studied for its wound healing associated biological activities both in vitro and in vivo, to the best of our knowledge for the first time. The GC-MS and GC-FID analyses revealed the abundance of the bioactive compounds, phytol, and 1-heptadecanol, which are also associated with the antioxidant and anti-inflammatory properties. The P. russeliana methanol extract showed moderate anti-Helicobacter pylori activity, LOX inhibitory and antioxidant activity. Following in vitro tests with anti-inflammatory and analgesic effects, expected supportive results were obtained in in vivo wound healing experiments. The anti-inflammatory and analgesic activity of $P$. russeliana extract was statistically significant, supported by both in vitro and in vivo wound healing activity, suggesting that Phlomis species can be correlated to traditional uses. The experimental design supports the wound 
healing properties by scientific evidence and the further potential of the ethnobotanical application of Phlomis species.

Supplementary Materials: The following are available online: Scheme S1: Bioactivity-Guided Fractionation and Chemical Characterization.

Author Contributions: Conceptualization, M.E.O., A.E.K., and F.D.; methodology, M.E.O., Ş.A., Y.Ö., B.D. (Benay Daylan), N.Ü.O. and H.S.; software, validation, M.E.O., Y.Ö., and H.S.; formal analysis, A.E.K. and B.D. (Benay Daylan); investigation, M.E.O., Y.Ö. and A.E.K.; resources, F.D. and B.D. (Betül Demirci); data curation, M.E.O.; writing—original draft preparation, M.E.O., A.E.K.; writing—review and editing, F.D.; visualization, Ş.A. and B.D. (Benay Daylan); supervision, F.D.; project administration, F.D. All authors have read and agreed to the published version of the manuscript.

Funding: This research received no external funding.

Acknowledgments: This research project was supported by Anadolu University Scientific Research Projects Commision (BAP 1807S259). The authors would like to thank to Muhammet Karadağ for the assistance in collecting the plant material and Şükran Kültür for identifying confirmation.

Conflicts of Interest: The authors declare no conflicts of interest.

\section{References}

1. Akaydin, G.; Şimşek, I.; Arituluk, Z.C.; Yeşilada, E. An ethnobotanical survey in selected towns of the mediterranean subregion (Turkey). Turkish J. Biol. 2013, 37, 230-247.

2. Amor, I.L.B.; Boubaker, J.; Sgaier, M.B.; Skandrani, I.; Bhouri, W.; Neffati, A.; Kilani, S.; Bouhlel, I.; Ghedira, K.; Chekir-Ghedira, L. Phytochemistry and biological activities of Phlomis species. J. Ethnopharmacol. 2009, 125, 183-202. [CrossRef] [PubMed]

3. Demirci, B.; Toyota, M.; Demirci, F.; Dadandi, M.Y.; Can Baser, K.H. Anticandidal pimaradiene diterpene from Phlomis essential oils. Comptes Rendus Chim. 2009, 12, 612-621. [CrossRef]

4. Demirci, F.; Guven, K.; Demirci, B.; Dadandi, M.Y.; Baser, K.H.C. Antibacterial activity of two Phlomis essential oils against food pathogens. Food Control 2008, 19, 1159-1164. [CrossRef]

5. Kırmızıbekmez, H.; Montoro, P.; Piacente, S.; Pizza, C.; Dönmez, A.; Çalış, İ. Identification by HPLC-PAD-MS and quantification by HPLC-PAD of phenylethanoid glycosides of five Phlomis species. Phytochem. Anal. 2005, 16, 1-6. [CrossRef]

6. ALpay, M.; Dulger, G.; Sahin, I.E.; Dulger, B. Evaluating antimicrobial and antioxidant capacity of endemic Phlomis russeliana from Turkey and its antiproliferative effect on Human Caco-2 Cell Lines. An. Acad. Bras. Cienc. 2019, 91, e20180404. [CrossRef]

7. Benbow, M. Wound care: Ensuring a holistic and collaborative assessment. Br. J. Community Nurs. 2011, 16, S6-S16. [CrossRef]

8. Haque, S.D.; Saha, S.K.; Salma, U.; Nishi, M.K.; Rahaman, M.S. Antibacterial Effect of Aloe vera (Aloe barbadensis) leaf gel against Staphylococcus aureus, Pseudomonas aeruginosa, Escherichia coli and Klebsiella pneumoniae. Mymensingh Med. J. 2019, 28, 490-496.

9. Amri, B.; Martino, E.; Vitulo, F.; Corana, F.; Kaâb, L.B.-B.; Rui, M.; Rossi, D.; Mori, M.; Rossi, S.; Collina, S. Marrubium vulgare L. Leave Extract: Phytochemical Composition, Antioxidant and Wound Healing Properties. Molecules 2017, 22, 1851. [CrossRef]

10. Sasidharan, S.; Nilawatyi, R.; Xavier, R.; Latha, L.Y.; Amala, R. Wound Healing Potential of Elaeis guineensis Jacq Leaves in an Infected Albino Rat Model. Molecules 2010, 15, 3186-3199. [CrossRef]

11. Tanrıverdi, S.T.; Suat, B.; Azizoğlu, E.; Köse, F.A.; Özer, Ö. In-vitro evaluation of dexpanthenol-loaded nanofiber mats for wound healing. Trop. J. Pharm. Res. 2018, 17, 387-394. [CrossRef]

12. Siafaka, P.I.; Zisi, A.P.; Exindari, M.K.; Karantas, I.D.; Bikiaris, D.N. Porous dressings of modified chitosan with poly(2-hydroxyethyl acrylate) for topical wound delivery of levofloxacin. Carbohydr. Polym. 2016, 143, 90-99. [CrossRef] [PubMed]

13. Tsala, D.; Nnanga, N.; Ndzana, M.; Mballa, B.; Dimo, T. Evaluation of the antioxidant activity and the healing action of the ethanol extract of Calotropis procera bark against surgical wounds. J. Intercult. Ethnopharmacol. 2015, 4, 64-69. [CrossRef] [PubMed] 
14. Lodhi, S.; Jain, A.P.; Sharma, V.K.; Singhai, A.K. Wound-Healing Effect of Flavonoid-Rich Fraction from Tephrosia purpurea Linn. on Streptozotocin-Induced Diabetic Rats. J. Herbs. Spices Med. Plants 2013, 19, 191-205. [CrossRef]

15. Tasdemir, D.; Dönmez, A.A.; Çalis, I.; Rüedi, P. Evaluation of biological activity of Turkish plants. Rapid screening for the antimicrobial, antioxidant, and acetylcholinesterase inhibitory potential by TLC bioautographic methods. Pharm. Biol. 2004, 42, 374-383. [CrossRef]

16. Turker, A.U.; Yildirim, A.B. Evaluation of antibacterial and antitumor activities of some Turkish endemic plants. Trop. J. Pharm. Res. 2013, 12, 1003-1010. [CrossRef]

17. Guimarães, F.R.; Sales-Campos, H.; Nardini, V.; da Costa, T.A.; Fonseca, M.T.C.; Júnior, V.R.; Sorgi, C.A.; da Silva, J.S.; Chica, J.E.L.; Faccioli, L.H.; et al. The inhibition of 5-Lipoxygenase (5-LO) products leukotriene B4 (LTB 4 ) and cysteinyl leukotrienes (cysLTs) modulates the inflammatory response and improves cutaneous wound healing. Clin. Immunol. 2018, 190, 74-83. [CrossRef]

18. Brogliato, A.R.; Moor, A.N.; Kesl, S.L.; Guilherme, R.F.; Georgii, J.L.; Peters-Golden, M.; Canetti, C.; Gould, L.J.; Benjamim, C.F. Critical Role of 5-Lipoxygenase and Heme Oxygenase-1 in Wound Healing. J. Investig. Dermatol. 2014, 134, 1436-1445. [CrossRef]

19. Demetzos, C.; Dimas, K.; Hatziantoniou, S.; Anastasaki, T.; Angelopoulou, D. Cytotoxic and Anti-Inflammatory Activity of Labdane and cis-Clerodane Type Diterpenes. Planta Med. 2001, 67, 614-618. [CrossRef]

20. Fernández, M.A.; Tornos, M.P.; García, M.D.; de las Heras, B.; Villar, A.M.; Sáenz, M.T. Anti-inflammatory activity of abietic acid, a diterpene isolated from Pimenta racemosa var. grissea. J. Pharm. Pharmacol. 2001, 53, 867-872. [CrossRef]

21. Demiray, H.; Tabanca, N.; Estep, A.S.; Becnel, J.J.; Demirci, B. Chemical composition of the essential oil and n-hexane extract of Stachys tmolea subsp. Tmolea Boiss., an endemic species of Turkey, and their mosquitocidal activity against dengue vector Aesdes aegypti. Saudi Pharm. J. 2019, 27, 877-881. [CrossRef] [PubMed]

22. Kıvçak, B.; Akay, S.; Demirci, B.; Başer, K. Pharmaceutical Biology Chemical Composition of Essential Oils from Leaves and Twigs of Pistacia lentiscus, Pistacia lentiscus var. chia, and Pistacia terebinthus from Turkey. Pharm. Biol. 2008, 42, 360-366. [CrossRef]

23. Gül, S.; Demirci, B.; Başer, K.H.C.; Akpulat, H.A.; Aksu, P. Chemical Composition and In Vitro Cytotoxic, Genotoxic Effects of Essential Oil from Urtica dioica L. Bull. Environ. Contam. Toxicol. 2012, 88, 666-671. [CrossRef] [PubMed]

24. Silva, R.O.; Sousa, F.B.M.; Damasceno, S.R.B.; Carvalho, N.S.; Silva, V.G.; Oliveira, F.R.M.A.; Sousa, D.P.; Aragão, K.S.; Barbosa, A.L.R.; Freitas, R.M.; et al. Phytol, a diterpene alcohol, inhibits the inflammatory response by reducing cytokine production and oxidative stress. Fundam. Clin. Pharmacol. 2014, 28, 455-464. [CrossRef]

25. Shi, G.B.; Wang, B.; Wu, Q.; Wang, T.C.; Wang, C.L.; Sun, X.H.; Zong, W.T.; Yan, M.; Zhao, Q.C.; Chen, Y.F.; et al. Evaluation of the wound-healing activity and anti-inflammatory activity of aqueous extracts from Acorus calamus L. Pak. J. Pharm. Sci. 2014, 27, 91-95.

26. Murthuza, S.; Manjunatha, B.K. In vitro and in vivo evaluation of anti-inflammatory potency of Mesua ferrea, Saraca asoca, Viscum album \& Anthocephalus cadamba in murine macrophages raw 264.7 cell lines and Wistar albino rats. Beni-Suef Univ. J. Basic Appl. Sci. 2018, 7, 719-723.

27. Bechert, K.; Abraham, S.E. Pain Management and Wound Care. J. Am. Col. Certif. Wound Spec. 2009, 1, 65-71. [CrossRef]

28. Chen, C.S.; Su, W.H.; Cheng, M.H.; Lee, W.L.; Tsou, T.S.; Chang, W.H.; Wang, P.H. Nonsteroidal anti-inflammatory drugs for wounds: Pain relief or excessive scar formation? Mediators Inflamm. 2010, 2010, 1-8. [CrossRef]

29. Gokce, E.H.; Tuncay Tanrıverdi, S.; Eroglu, I.; Tsapis, N.; Gokce, G.; Tekmen, I.; Fattal, E.; Ozer, O. Wound healing effects of collagen-laminin dermal matrix impregnated with resveratrol loaded hyaluronic acid-DPPC microparticles in diabetic rats. Eur. J. Pharm. Biopharm. 2017, 119, 17-27. [CrossRef]

30. Okur, M.E.; Karantas, I.D.; Şenyiğit, Z.; Okur, N.Ü.; Siafaka, P.I. Recent trends on wound management; new therapeutic choices based on polymeric carriers. Asian J. Pharm. Sci. 2020. [CrossRef]

31. Rangaraj, A.; Harding, K.; Leaper, D. Role of collagen in wound management. Wounds UK 2011, 7, 54-63.

32. Han, G.; Ceilley, R. Chronic Wound Healing: A Review of Current Management and Treatments. Adv. Ther. 2017, 34, 599-610. [CrossRef] [PubMed] 
33. Ayla, Ş.; Günal, M.Y.; Sayin Şakul, A.; Biçeroğlu, Ö.; Özdemir, M.E.; Okur, M.E.; Çiçek Polat, D.; Üstündağ Okur, N.; Bilgiç, B.E. Effects of Prunus spinosa L. fruits on experimental wound healing. Medeni. Med. J. 2017, 32, 152-158. [CrossRef]

34. Oda, Y.; Kagami, H.; Ueda, M. Accelerating Effects of Basic Fibroblast Growth Factor on Wound Healing of Rat Palatal Mucosa. J. Oral Maxillofac. Surg. 2004, 62, 73-80. [CrossRef]

35. Blois, M.S. Antioxidant determinations by the use of a stable free radical. Nature 1958, 181, 1199-1200. [CrossRef]

36. Re, R.; Pellegrini, N.; Proteggente, A.; Pannala, A.; Yang, M.; Rice-Evans, C. Antioxidant activity applying an improved ABTS radical cation decolorization assay. Free Radic. Biol. Med. 1999, 26, 1231-1237. [CrossRef]

37. Spanos, G.A.; Wrolstad, R.E. Influence of processing and storage on the phenolic composition of Thompson Seedless grape juice. J. Agric. Food Chem. 1990, 38, 1565-1571. [CrossRef]

38. Karadağ, A.E.; Demirci, B.; Çaşkurlu, A.; Demirci, F.; Okur, M.E.; Orak, D.; Sipahi, H.; Başer, K.H.C. In vitro antibacterial, antioxidant, anti-inflammatory and analgesic evaluation of Rosmarinus officinalis L. flower extract fractions. S. Afr. J. Bot. 2019, 125, 214-220. [CrossRef]

39. Whitmire, J.M.; Merrell, D.S. Successful Culture Techniques for Helicobacter Species: General Culture Techniques for Helicobacter pylori. Adv. Struct. Saf. Stud. 2012, 921, 17-27. [CrossRef]

40. Baylac, S.; Racine, P. Inhibition of 5-lipoxygenase by essential oils and other natural fragment extracts. Int. J. Aromather. 2003, 13, 138-142. [CrossRef]

41. Aydin, A.; Reis, R.; Sipahi, H.; Zeybekoğlu, G.; Çelik, N.; Kırmızıbekmez, H.; Kaklıkkaya, N. Hydroxytyrosol: The Phytochemical Responsible for Bioactivity of Traditionally used Olive Pits. Euroasian J. Hepato-Gastroenterol. 2018, 8, 126-132. [CrossRef] [PubMed]

42. Kırmızıbekmez, H.; İnan, Y.; Reis, R.; Sipahi, H.; Gören, A.C.; Yeşilada, E. Phenolic compounds from the aerial parts of Clematis viticella L. and their in vitro anti-inflammatory activities. Nat. Prod. Res. 2019, 33, 2541-2544. [CrossRef] [PubMed]

43. Galeano, M.; Altavilla, D.; Bitto, A.; Minutoli, L.; Calò, M.; Cascio, P.L.; Polito, F.; Giugliano, G.; Squadrito, G.; Mioni, C.; et al. Recombinant human erythropoietin improves angiogenesis and wound healing in experimental burn wounds *. Crit. Care Med. 2006, 34, 1139-1146. [CrossRef] [PubMed]

Sample Availability: Samples are available from the authors. 